\title{
Dynamic deformation evolution of the adiabatic shear bands in zirconium alloy formed at a strain rate of about $2300 \mathrm{~s}^{-1}$
}

\author{
Dongli Zou*, Dawu Xiao, Chao Lu, Lifeng He and Yawen Zhao \\ Institute of Materials, China Academy of Engineering Physics, 621907 Jiang You, China
}

\begin{abstract}
Deformed microstructure of the adiabatic shear bands in zirconium alloy impacted by split Hopkinson pressure bar at a strain rate of about $2300 \mathrm{~s}^{-1}$ was characterized systemically. Four different strains of zirconium alloy subjected to impact loading at same strain rate were designed by means of strain stopper rings. At a strain of 0.30 , the transformed bands were distinguished at the tip of crack. The transformed bands mainly composed of the ultrafine and equiaxed grains with the mean diameter of about 100 300 nm were found by a dual beam FIB/TEM system. Besides ultrafine grains in the transformed bands, nanometer fine grain was observed and confirmed by HRTEM. Thus, the transformed bands mainly composed of the mixed microstructure including nanometer and ultrafine grains were confirmed, and no phase transformation and amorphization in transformed bands take place.
\end{abstract}

\section{Introduction}

Zirconium alloys as the fuel cladding material have been widely used in the nuclear power reactors because of the high melting point, low neutron absorption cross section, good corrosion resistance and excellent mechanical properties [1]. Broad applications of zirconium alloys lead to an increasing tendency that they would be subjected to the dynamic loading or high strain rate loading inevitably. Thus, it is necessary to achieve fundamental understanding on the dynamic deformation behaviors of zirconium alloy subjected to high strain rate loading.

Adiabatic shear bands (ASBs) are considered as an important fracture mode of materials under dynamic deformation, and the ASBs have received much attention due to the commonly accepted fact that ASBs are regarded as the precursor of materials damage and failure under dynamic deformation. Based on the optical morphologies and microstructural difference, two types of ASBs have been classified [2, 3]: deformed bands (DBs) and transformed bands (TBs). However, only several references about microstructural characteristics of ASBs in zirconium alloy have been reported in recent years. The ultrafine and equiaxed grains in the ASBs characterized by electron backscatter diffraction (EBSD) have been reported by us [4, 5], and beside the ultrafine and equiaxed grains, other microsstructural characteristics in TBs were not detected due to the spatial resolution limit of EBSD technology. Wang et al. $[6,7]$ investigated the microstructural characteristics of ASBs in zirconium alloy under dynamic deformation. Besides the ultrfine grains, the nanostructural grains in ASBs was found, showing that the microstructure in the ASBs was mainly composed of ultrafine and nanostructured composite grains. In this paper, the microstructure of ASBs in zirconium alloy is characterized by means of extensive microscopy, and the focused on beam (FIB) technology, due to its ability to perform precision in-situ milling, has been used to prepare characterization sample.

\section{Experimental}

The annealed zirconium alloy plate with thickness of 4.6 $\mathrm{mm}$ was selected, and the strong basal texture with basal poles paralleled to ND (normal direction) spreading \pm 20 to $40^{\circ}$ toward TD (transverse direction) was confirmed. The cylindrical specimens were cut from zirconium alloy plates with the $\mathrm{c}$ axis paralleled to ND, and diameter and height of the cylindrical specimens were $6 \mathrm{~mm}$ and 4.6 $\mathrm{mm}$, respectively. Dynamic compressive experiments were conducted on a split Hopkinson pressure bar (SHPB) in China Academy of Engineering Physics. After impact, the specimens were cut by cutting machine along the longitudinal direction, polished and finally etched by $45 \mathrm{ml} \mathrm{H}_{2} \mathrm{O}+45 \mathrm{ml} \mathrm{HNO}_{3}+10 \mathrm{ml} \mathrm{HF}$. The montage macroscopic views and metallographic microstructure of the specimens were observed by a CLSM (MRC-1000) and a SEM (KYKY-1010B). Electron backscatter diffraction (EBSD) specimens were prepared by electro-polishing in a solution: $70 \mathrm{ml}$ $\mathrm{CH}_{3} \mathrm{OH}+20 \mathrm{ml} \mathrm{C}_{6} \mathrm{H}_{14} \mathrm{O}_{2}+10 \mathrm{ml} \mathrm{HClO}_{4}$, and the polishing voltage and temperature were kept at $20 \mathrm{~V}$ and $-30{ }^{\circ} \mathrm{C}$, respectively. EBSD tests were conducted on a high resolution FESEM (FEI Helios nanolab 600i) with a TSL system operated at $25 \mathrm{kV}$. A dual beam FIB (FEI Helios NanoLab DualBeam system) was used to prepare the TEM specimens in ASBs area, and TEM observation

* Corresponding author: dlzou1980@163.com 
was carried out on FEI Titan 80-300 spherical aberration electron microscope operated at $300 \mathrm{KV}$.

\section{Results and discussion}

Fig. 1 shows the stress-strain curves of zirconium alloy impacted by a SHPB at a strain rate of $2300 \mathrm{~s}^{-1}$. Four strain stopper rings with different heights are used to control the strain, leading to four strain levels obtained at same strain rate. Four strain stopper rings with the heights $0.50 \mathrm{~mm}, 0.90 \mathrm{~mm}, 1.20 \mathrm{~mm}$ and $1.30 \mathrm{~mm}$ are used, respectively, leading to four true strains $0.11,0.21$, 0.30 and 0.33 obtained. The obtained four strain levels have been drawn in Fig .1. The mean strain rates are calculated from the strain rate-time curves, and the calculated mean strain rate values from four dynamic compressive experiments are almost same, about 2300 $\mathrm{s}^{-1}$, indicating that the strain is the only parameter to influence the dynamic fracture process.

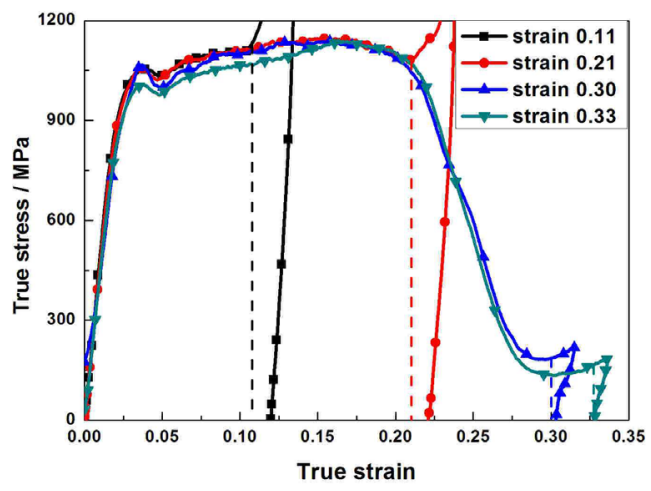

Fig. 1. Stress-strain curve of zirconium alloy impacted by a SHPB at a strain rate of $2300 \mathrm{~s}^{-1}$.

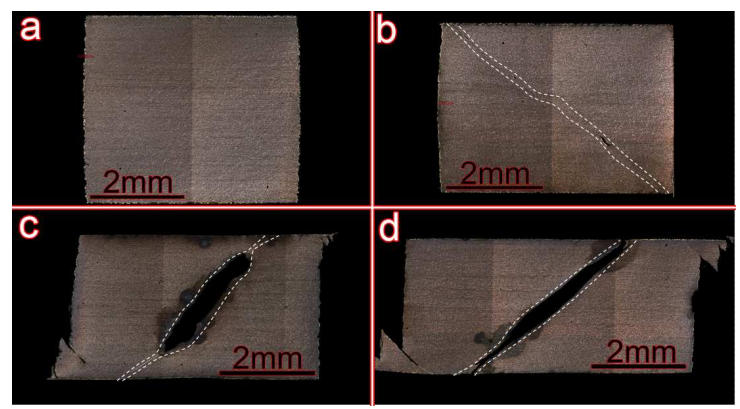

Fig. 2. macroscopic images of zirconium alloy deformed a stain rate of about $2300 \mathrm{~s}^{-1}$ : (a) stain 0.11 , (b) strain 0.21 , (c) strain 0.30 , (d) strain 0.33 .

The corresponding macroscopic images of the specimens deformed at four strain levels can be seen in Fig. 2. At a strain of about 0.11 , almost no strain localizations, micro-voids or micro-cracks are found on specimen cross-section view. At a strain of about 0.21, the DBs crossing through the specimen from one corner $45^{\circ}$ to another corner are observed, while a few microvoids and micro-cracks in the DBs are observed. With the strain increasing to 0.31 , the macro-crack and TBs at the tip of the crack are observed on specimen crosssection view, and the propagated direction of the macrocrack is about $45^{\circ}$ to the dynamic compression direction.
At a strain of 0.33 , the macro-crack crossing the whole specimen is observed, and the propagated direction is $45^{\circ}$ to impacted direction. The trace of the TBs at the rim of macro-cracks can be seen, showing that the TBs have been formed before macro-cracks formation.

At a strain of 0.30 and a strain rate of $2300 \mathrm{~s}^{-1}$, typical TBs formed in zirconium alloy are observed and confirmed, as shown in Fig. 3. The TBs and macro-crack propagated about $45^{\circ}$ or $135^{\circ}$ to impact direction can be observed in Fig .3a, which should be associated with the maximum shear stress direction. The TBs are located at two tips of macro-crack, and the corresponding high magnified CLSM image of Fig. 3a inserted zone B is shown in Fig. 3b. The TBs are appeared as a distinct narrow band, and the boundary between the narrow band and the matrix is distinct. Almost no transition zone from the TBs to the matrix can be found. Meanwhile, a few micro-cracks formed in the TBs can be observed in Fig. $3 \mathrm{~b}$, and the propagated direction of the micro-crack is consistent with that of TBs, indicating that TBs can be acted as preferential sites for micro-cracks initiation and propagation.

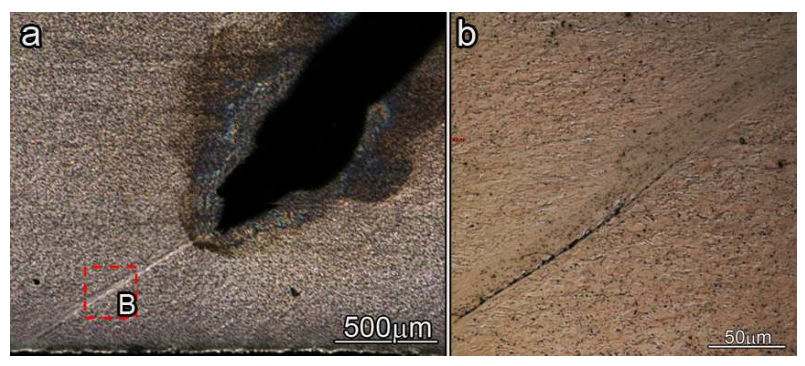

Fig. 3. CLSM images of TBs in zirconium alloy deformed at a strain of 0.30 and a strain rate of $2300 \mathrm{~s}^{-1}$ : (a) macroscopic CLSM images of TBs, (b) high magnified CLSM image of TBs.

EBSD indexed and post-processed images of the TBs formed in zirconium alloy deformed at a strain of 0.30 and a strain rate of $2300 \mathrm{~s}^{-1}$ are shown in Fig. 4 . The EBSD indexed zone is a combined image of the TBs and neighbor matrix, and the TBs are located at the center of the indexed zone. The boundary between the matrix and the TBs is distinct, as shown in Fig. 4a, and almost no transition zone from the neighbor matrix to the TBs can be found. The grain size in the center of the TBs decrease several orders of magnitude and the grains with diameter less than one hundred nanometers can not be detected because of the limitation of the SEM resolution rate. The corresponding post-processed band contrast images of the indexed zone in Fig. 4a are shown in Fig. $4 \mathrm{~b}$, and high and low angle boundaries inserted in the grain boundary image are shown in Fig. 4c. The gradient transition of the grain size from TBs rim to the center of the TBs can be distinguished from the EBSD image, which should be associated with temperature distribution in the TB. The gradient temperature distribution and the maximum temperature rising at the center of the ASBs lead to the grains at the center of the TBs finer than the adjacent zones. Compared with original grains with diameter ranging from 10 to $20 \mu \mathrm{m}$, the grain size in the center of the TBs has decreased about two orders of 
magnitude. However, the statistic distribution of the real grain size in the center of the TBs is difficult to obtain due to much lower indexed rate in TBs. The density of the low angle boundary (misorientation between $2^{\circ}$ and $15^{\circ}$ ) in the grains in the TBs is much lower than that of the neighbor matrix, indicating that the dislocation or defect density has decreased during the formation of the ultrafine and equiaxed grain in the TBs.

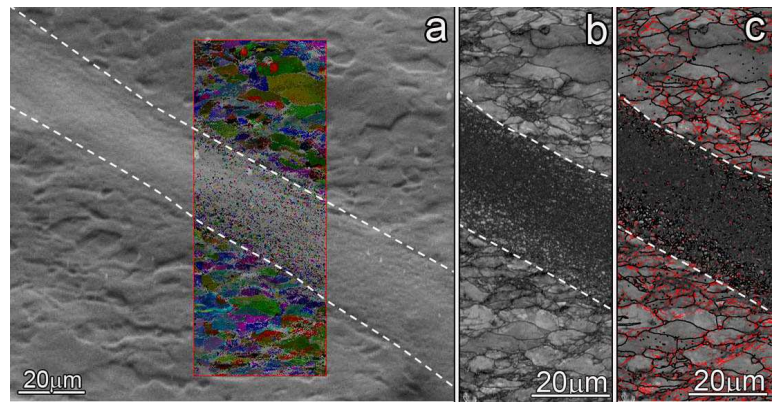

Fig. 4. EBSD images of TBs in zirconium alloy deformed at a strain of 0.30 and a strain rate of $2300 \mathrm{~s}^{-1}$ : (a) indexed zone of TBs, (b) band contrast image of TB, (c) grain boundary image of TB. High and low angle boundaries are depicted as black and red lines.

A dual beam FIB, associated with microobservation and in-situ milling ability, is used to prepare TEM specimens in TBs area for microstructural characterization, and the detail preparation process can be seen in Fig. 5. It includes several procedures: selected position, ion-milling, jointing and ion-thinning. Firstly, the TEM observed zone of TBs should be located in SEM image, and the selected zone is cut by the ionmilling. Secondly, the cut sample is transferred from matrix to copper net by the manipulator, as shown in Fig. $5 \mathrm{~b}$ and $5 \mathrm{~d}$. Final thinning to electron transparency is achieved by ion-milling on copper net, and the samples with a thickness of about 50 100 nm are used to TEM observation.

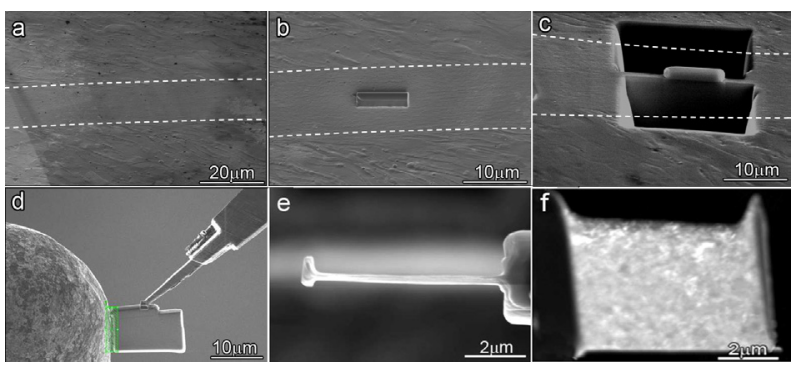

Fig. 5. TEM sample preparation of the microstructure in TBs formed at a strain of 0.30 and a strain rate of $2300 \mathrm{~s}^{1}$ : (a) SEM image, (b) deposition Pt, (c) ion-milling, (d) sample transferred by manipulator, (e) TEM sample, (f) TEM image.

TEM images of the deformed microstructure in TBs are shown in Fig. 6. TEM sample composed of sandwich structure can be seen in Fig. 6a, including platinum deposition layer, Oxidation amorphization layer and deformed microstructure. It can be seen that the TBs are mainly composed of the ultrafine and equiaxed grains, and mean diameter of the grains is about $100 \sim 300 \mathrm{~nm}$. The selected-area electron diffraction (SAED) of the deformed microstructure in TBs shows the hexagonal close-packed (hcp) Zr grains. No other crystal structure $\mathrm{Zr}$ grain and amorphous microstructure in $\mathrm{TBs}$ are detected, indicating that the phase transformation and amorphization in TBs not take place. Besides ultrafine grains in the bands, nanometer fine grain is observed and confirmed by HRTEM, as shown in Fig.7, and lots of defects in nanometer grains are found. Thus, the mixed microstructure composed of nanometer and ultrafine grains in TBs is formed in zirconium alloy.

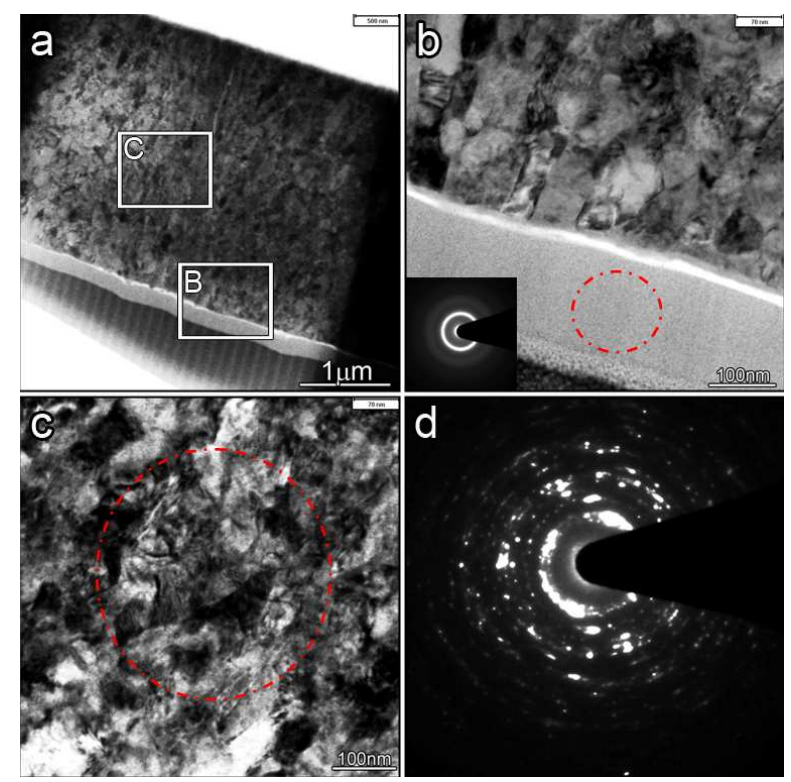

Fig. 6. TEM images of the deformed microstructure in transformed bands formed at a strain of 0.30 and a strain rate of $2300 \mathrm{~s}^{-1}$ : (a) low magnified image; (b) high magnified image in Fig. 6a and zone B; (c) high magnified image in Fig. 6a and zone $\mathrm{C}$; (d) SAED in Fig. $6 \mathrm{c}$.

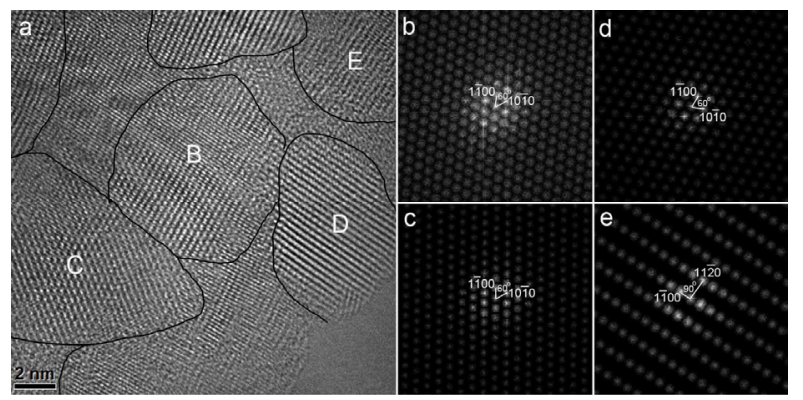

Fig. 7. HRTEM images of the deformed microstructure in transformed bands formed at a strain of 0.30 and a strain rate of $2300 \mathrm{~s}^{-1}$ : (a) (a) HRTEM; (b) SAED in zone B; (c) SAED in zone C; (d) SAED in zone D; (e) SAED in zone E.

\section{Conclusions}

The deformed microstructure of ASBs in zirconium alloy is characterized, and the following conclusions are drawn:

1. Four different strain levels of zirconium alloy subjected to dynamic deformation at same strain rate are designed by strain stopper rings, and four stain values are $0.11,0.21,0.30$ and 0.33 , respectively. 
2. At the strain of 0.30 , the TBs in the zirconium alloy are found, and the transformed bands mainly composed of the mixed microstructure including nanometer and ultrafine grains were confirmed.

Thanks for the support of National Natural Science Foundation (51401187).

\section{References}

1. D.L. Zou, B.F. Luan, Q. Liu, Trans. Nonferrous Met. Soc. China, 22 (2012)

2. Y.B. Xu, Y.L. Bai, M.A. Meyers, J. Mater. Sci. Technol., 22 (2006)
3. L. Zhen, D.L. Zou, C.Y. Xu, W.Z. Shao, Mater. Sci. Eng. A, 527 (2010)

4. D.L. Zou, B.F. Luan, Q. Liu, L.J. Chai, J.W. Chen, J. Nucl. Mater., 437 (2013)

5. D.L. Zou, B.F. Luan, Q. Liu, L.J. Chai, J.W. Chen, Mater. Sci. Eng. A, 558 (2012)

6. S.F. Li, W.K. Liu, A.J. Rosakis, T. Belytschko, W. Hao, Inter. J. Solids Struct., 39 (2002)

7. P.R. Guduru, A.J. Rosakis, G. Ravichandran, Mech. Mater., 33 (2001) 